\title{
Meaning Coconstruction in Group Counseling: The Development of Innovative Moments
}

\author{
Giovanna Esposito \\ SInAPSi Center, University of Naples, Federico II, Naples, Italy \\ Antonio P. Ribeiro, Daniela Alves, and Miguel M. Gonçalves 6 \\ School of Psychology, University of Minho, Braga, Portugal \\ Maria Francesca Freda \\ Department of Humanities, University of Naples Federico II, Naples, Italy
}

\begin{abstract}
This study discusses a model of group counseling, the narrative mediation path (NMP), which is a unique narrative, multimodal approach that combines four narrative modes (metaphoric, iconographic, writing, and bodily) and the narrative group. The purpose of the NMP is to foster reflexive processes with underachieving university students and to improve their academic performance. The study analyzes a single case of group counseling for seven underachieving economics students at an Italian university and the process of meaning construction among NMP narrative modes and the follow-up session. It applies the innovative moments coding system, a reliable method for studying change by tracking narrative innovations in sessions. More specifically, we focus on how innovative moments (IMs: action, reflection, protest, and reconceptualization) evolve during the four different narrative modes and the follow-up session. The findings suggest that the NMP fosters narrative innovations, mainly of a reflexive nature (reflection and reconceptualization). Moreover, during counseling and the follow-up session, the pattern of change is primarily characterized by reconceptualization IMs, the most complex form of narrative innovation.
\end{abstract}

This study stems from the need to develop novel intervention models to deal with underachievement in higher education, a phenomenon currently hampering the processes of inclusion and participation of many students in universities. In recent years, underachievement in university students has become a growing issue, with $35 \%$ of European students at risk of dropping out due to their academic performance.

Underachievement is commonly seen as a discrepancy between the level of students' performance and their academic potential. Such discrepancy must not be the direct result of a diagnosed learning disability and it must persist over an extended period of time (Reis \& McCoach, 2000). Many scholars agree that there is not a single cause of underachievement; instead, it is

Received 2 March 2016; accepted 20 August 2016.

Address correspondence to Giovanna Esposito, SInAPSi Center, University of Naples, Federico II, Naples, Italy. E-mail: giovan.esposito@unina.it 
influenced by numerous factors, including family problems, teaching methods, personality traits, and impairments in relational or reflexive competences (e.g., Baslanti \& McCoach, 2006; Rimm, 2003).

The European Commission has called for the development and implementation of innovative approaches, such as guidance and counseling, to help underachieving university students fully reap the benefits of tertiary education and prevent their academic exclusion. Moreover, such approaches entail helping individuals overcome barriers to accessing, participating in, and completing higher education. According to the Council of the European Union (2013), a crucial element for increasing students' participation and inclusion is the provision of tailored guidance that can help students make informed decisions, which, in turn, can reduce the dropout rate.

To respond to these needs and challenges, we developed and implemented the Innovative Solution to Acquire Learning to Learn (INSTALL) European Project, ${ }^{1}$ which involved 198 underachieving students enrolled in different European university degree programs. INSTALL was founded in 2011 within the ERASMUS Multilateral Project Measure, specifically addressed to those nonconventional student populations (underachieving, nontraditional/disadvantaged, immigrants, first-generation students, etc.) who might experience difficulties in successfully graduating from university. The INSTALL Project was completed in 2014, and the Center for Active and Integrated Inclusion of Students (SInAPSi) from the Federico II University of Naples (Italy) took the lead in the project partnership with three other European universities: University of Seville (US), National University of Ireland Maynooth (NUIM), National School of Political Studies and Public Administration (NSPSA). Specifically, INSTALL was developed and implemented in order to promote Learning to Learn (L2L) Key Competence with underachieving university students. The $\mathrm{L} 2 \mathrm{~L}$ is conceived as the outcome of a reflexive process, which allows the students to recognize, process, and adjust their thoughts, beliefs, and emotions within the entire learning process. The project stemmed from the hypothesis, as documented in literature (e.g., Twemlow, Fonagy, \& Sacco, 2005), that, through the development of reflexive processes, underachieving students could understand how their taken-for-granted meanings of their university experience affected the achievement of their learning goals.

For the purposes of the INSTALL Project, Esposito and Freda (2016) developed the narrative mediation path (NMP), a novel narrative counseling model aimed at improving students' academic performance, which is considered the main indicator of $\mathrm{L} 2 \mathrm{~L}$ and academic inclusion (e.g., Bacchini, Esposito, \& Affuso, 2009; Muskens, 2011). Specifically, NMP was implemented in order to enhance students' reflexive processes, as there is a close relationship between good academic performance and reflexive competence (e.g., Taylor, 2011), and the latter may be useful for encouraging critical thinking and supporting students' academic productivity (Esposito, Freda, \& Bosco, 2015).

Generally, reflexive processes can be defined as the mental processes that allow individuals to achieve knowledge by refocusing thinking on the action itself. Because this process has been a controversial subject in psychology, pedagogy, and philosophy, various definitions have been proposed over the years (e.g., Kember, McKay, Sinclair, \& Wong, 2008; Moore, 2011) and many models of the reflexive process have been developed (e.g., Bleakley, 2006).

Recently, Esposito and Freda (2016) proposed a socio-constructivist conceptualization that views the reflexive process as a multidimensional and intersubjective product, and conceives it in terms of innovations in the meaning system of subjects. In this regard, two levels of different 
complexity were distinguished: (a) reflection, which is the psychological process whereby individuals are able to gain a new understanding of the self (e.g., "That day during class, I understood that I was an incapable student, and I did not know that before"); and (b) reflexivity, which is the ability to think about the reasons of one's behavior and thus recognize the active role the subjects assume in guiding their relationship with the overall context (e.g., "Today I realized that I deliberately slowed down in my second year at the university to help my twin sister catch up with me").

Moreover, according to this conceptualization, a reflexive process cannot be confined to individual minds, but is a process of meaning coconstruction between subjects involved in a shared context. In other words, each member of the relationship allows for multiple viewpoints of the experience (i.e., one listens, considers, and elaborates), allowing the subject to provide new meaning to the experience as well as to enhance the reflexive meaning construction of this experience (Salvatore, 2011). Following this conceptualization, assuming a reflexive position on their own university experience allows students to recognize their own limitations and resources to strategically perform in their coursework (Esposito \& Freda, 2015). Underachieving students often have reflexive difficulties when they face developmental tasks (e.g., failing an exam) and consequent problems using their time strategically (Padykula \& Horwitz, 2011). Students' reflexive impairments influence their sense of agency - namely, their ability to act as intentional beings at the university (Caston, 2011). It is assumed that an increase in reflexive processes equates with the ability to transform one's situation by enhancing one's ability to make choices and act in a goal-oriented manner.

The present study includes a pilot study that analyzes a single case of group counseling for seven underachieving students at an Italian university and the process of meaning construction during the NMP narrative modes. Specifically, it analyzes the process of meaning construction during the NMP counseling intervention and in the follow-up session by using the innovative moments coding system (IMCS; Gonçalves, Ribeiro, Mendes, Matos, \& Santos, 2011; Gonçalves, Ribeiro, Mendes, Alves et al., in press), which is a reliable method for studying change by tracking narrative innovations.

This study is organized into three main sections. First, we present the NMP model of counseling intervention and its role in improving the reflexive process. Then, we introduce the pilot study that analyzes the process of meaning construction in one group counseling, which includes the seven Italian university students. Finally, we discuss the implications of these findings for NMP counseling research and practice.

\section{The Group Counseling Method: The NMP}

The relevance of narrative approaches in counseling is not new, and many narrative models have been developed (e.g., Savickas, 2005) that focus on various problems and integrate different counseling intervention settings (Richardson, 2012). According to Murdock, Dual, and Nilsonn (2012), the narrative approach to counseling presents a well-defined theoretical structure supported by a considerable body of empirical research. In addition, it is consistent with the values and philosophy of counseling psychology. Starting from these assumptions, within the INSTALL Project, a narrative model of group counseling was developed to foster reflexive processes with underachieving university students to positively impact their academic performance and increase their agency at the university. 
According to this counseling narrative model, narratives play a key role in developing reflexive processes by allowing individuals to construct meaning to their experience and reinterpret it from a different, more coherent perspective (Freda, Dicé, Auricchio, Salerno, \& Valerio, 2014). Specifically, reflexive processes draw on narrative structures to facilitate a representation of the events in one's own life (Lieblich, 2012).

The counseling approach adopted here made use of a narrative multimodal approach, conceived as a new form in which meaning is produced through the interrelationships between and among different media and modes (Dicks, Soyinka, \& Coffey, 2006). According to some scholars (e.g., Kress, 2005), multimodality may enhance the reflexive construction of meaning, as it promotes an understanding of the complexities of the experiences and allows for an in-depth analysis that would not have been possible through the use of a single narrative medium. Adopting a multimodal approach means using and amplifying the potential reflexive role of each narrative medium by introducing various narrative media through which subjects can perceive themselves and give new meaning to their experience.

In order to reach reflexive objectives, we developed the NMP, a seven-session (weekly) narrative multimodal approach that integrates group and individual narrative levels as well as four narrative modes: metaphoric, iconographic, writing, and bodily. Each mode uses its own medium. For example, the metaphoric mode (first and second sessions) involves maxims and proverbs, and the student is asked to choose one that represents his or her university experience (e.g., "He who makes his bed must lie in it" and, "Yes, we can!"). During the first two sessions, the counselor started a group discussion focused on the reasons behind the choices of the proverbs and mottos that each student had made, as well as the connections between their representation of "being a student" and of "doing" certain university tasks. The counselor provided feedback to the members of the group about the most common representations of what it meant to be a student as these emerged from the metaphors. For example, one student selected "He who makes his bed must lie in it" and augmented his choice by affirming that it depicted him as a victim of external factors (bad luck, ill will of certain professors, etc.). The counselor collected other group comments and invited the participants to think about the outcome of having adopted similar passive and dependent relational modalities in past events and their relationships at university, particularly with professors and colleagues.

Summarizing, metaphorical media presents an early opportunity to gain access to the representation of one's university experience and to give new meaning to it. Proverbs and maxims are "synchronous and summarized" (Radman, 1997) narrative media that allow students to develop a strong link to the cultural and generic connotation of their formative self.

In the iconographic mode (third and fourth sessions), six vignettes were shown, each featuring a character engaged in a typical situation of university life (e.g., enrollment, home study). In this case, the student was asked to choose one and create a balloon that includes the character's thoughts or feelings. Then, the counselor activated a group discussion and asked each student to read the six narrations to create a story. This helped the students identify their thoughts and emotions and to use the vignettes to give a coherent meaning to what they had written. For example, invited by the counselor to read and comment on the vignettes, a female student said that in all the vignettes she choose a protagonist in a central position, and from this position she could speak to everyone and make several questions to her mates. Other members of the group commented that the student seemed to be involved in simply "causal questions" unrelated to university tasks. These comments gave the student the opportunity to reflect on her propensity 
to waste time at university and on her way in relating to mates. In light of the feedback from the other group members, the counselor promoted group discussion on how "wasting time" could be a symptom of students' difficulties in involving in productive relationships at university. So, the vignettes invited students to identify themselves with a hypothetically chosen character. Moreover, the vignettes had a projective nature, as they requested students to represent their university experiences and, in doing so, made students feel less vulnerable and more in control (Shaw, 2002).

In the writing mode (fifth and sixth sessions), three narrative assignments are given: writing about a low point, a high point, and a decisional turning point in the student's university experience in accordance with McAdams's narrative model (McAdams et al., 2006). The counselor asked each participant to read the written assignments and involve group members by asking them what they thought and felt while listening to the other narrations. The counselor underlined the links between the low point, high point, and decisional turning point in order to analyze students' limitations and resources, such as to strengthen the decisional position assumed by students in the writings. For example, a male student wrote on the low point about his difficulty in asking questions to professors during class while in the high and the decisional point talked about his new ability in being more active in group discussions with peers and professors. The group discussion allowed the student to reinterpret his role and provide a representation of himself as an insecure student, with some limitations but also with some strengths. The counselor reflected on how this insecurity was often shown by the student even in the INSTALL setting and underlined his difficulty in participating and commenting on other members' narratives during the previous modes. The counselor then extended the group narration toward an understanding of how the more active role assumed by the student and other group members may be conceived as an expression of their more agentic stance during the intervention. As noted, different from the previous modes, writing explicitly invited students to narrate their previous actions and assume a more subjective position - namely, to be both the subject and the object of their narration. In the written narratives, students were asked to reflect on previous actions and to recognize the roles such actions assumed in orienting their university experience.

In the bodily mode (seventh session), students were asked to create a sculpture about their university future using the bodies of all of the participants. Sculpture is a creative, dynamic technique of intervention developed in the field of systemic psychotherapy (e.g., Vallario, 2012). Originally, its aim was to represent relationships within families or systems in a specific time and context. However, through the years, different types of sculptures have been developed (e.g., group and couples). In this counseling intervention, the sculpture approach was adopted to foster students' representation as subjects acting toward a particular goal. Through this approach, the students could represent the body in action, which may lead to a more goal-oriented practice. Specifically, the counselor invited the group to organize the sculpture by deciding how to place themselves and also on the facial expressions of all members. Then, the group was asked to remain still for a few seconds. After finishing the human sculpture, a photograph was taken. At the end of the sculpture, all students were asked to describe their emotions about the representation, their role in the sculpting, the difficulties they encountered, and their idea of their university future. This allowed students to identify themselves as belonging to a shared group representation; to recognize the fundamental role of group intersubjectivity in the development of their reflective competence; and, at the same time, to reveal significant aspects of their individual university experience. For example, one student sculpted a tree trunk, commenting that he realized how this 
may have been an expression of his rigidity and stiffness in trying (unsuccessfully) to organizing every aspect of his studying. However, he realized the trunk could also mean strength and that his colleagues had already recognized this aspect in him as they affirmed that he had gradually reduced his tendency to be rigid and moralistic with everyone along the sessions. This gave him the opportunity to plan to become a sort of "flexible branch" rather than a trunk.

Finally, a 2-hour follow-up session was carried out approximately one year after the end of the counseling. During the follow-up session, the students were asked to reflect on what had changed during the previous year. In addition, they were asked questions on how their participation in the group counseling contributed to the changes.

As noted, the sequence modes were presented in a way that favored the emergence of different representations of the formative self, such as becoming increasingly sharp, contextualized, and goal-oriented. Moreover, during each session (including the follow-up session), a four-step methodological sequence was followed: (a) narrative medium (each student was asked to chose or to fill in the narrative medium or to narrate the year's events); (b) narrative construction of the experience (starting from the medium, each group member narrated his or her university experience and was asked to give meaning to it); (c) group-level narrative meta-discourse (this was based on each member's narration); and (d) reconstruction of the narrative experience (each student was asked to elaborate a new meaning based on the previous individual and group narrative meta-discourse; Freda \& Esposito, in press).

\section{The NMP Counselor}

The NMP counselor is considered a methodological moderator who holds students' experiences and contributes to the coconstruction of meaning in the intersubjective group context. Because the counselor adopts a process-oriented approach, he or she focuses the group's attention on what happens in the here and now of the session. The focus is more on relationships than contents, and on the possibility of orienting the group discourse towards reflection/reflexivity. As noted, the group counseling includes complex objectives that need to be achieved in a limited amount of time (seven sessions). Thus, the counselor must adopt a "directive" approach, orienting and reinforcing the narrative innovations that emerge during the sessions, and assuming different roles and functions throughout the process. For example, in the beginning, the counselor's role is primarily aimed at broadening the discussion to involve each student, to collect and articulate the themes narrated through the different media, and to create a shared context. During the iconographic mode, the counselor attempts to connect the different narrative texts presented in the vignettes to identify a common narrative theme and analyze the actions performed by the students in various university situations. During the writing mode, the counselor's interventions generally decrease and become more interpretive. Moreover, the counselor mainly focuses on the students' resources and limits at the university, to allow them to conduct a realistic analysis of their area of potential expertise. During the bodily mode, the counselor shares the findings of the completed group work and progresses to more complex interventions with the goal of enabling students to plan their university futures.

When necessary, the counselor uses interpretations to enhance the ongoing meaning processes within the setting. In addition, he or she adopts balanced group turn-taking interactions and opens each session to building bridges with the previous one to create a sense of continuity and strengthen the narrative innovations. The purpose is to orient the group discourse toward 
university-related goals in such a way that the NMP counselor first allows each student to narrate his or her experiences and then identifies and discusses any redundancies in his or her way of feeling, thinking, and acting in different contexts. The final goal is to allow the students to reflect on the roles that are shaping their university underachievement (Freda, Gonzàlez-Monteagudo, \& Esposito, 2016).

\section{The IMCS}

From a narrative perspective, the interpretation and organization of life experiences entail a dynamic process through which the plurality of events become interconnected and articulated in a coherent meaning system (Adler, Skalina, \& McAdams, 2008; Angus, Levitt, \& Hardtke, 1999). As a result of this selective process, the dominant meaning system might not incorporate important aspects of individuals' lives that may play an important role in their self-reconstruction (Dimaggio, 2006; White \& Epston, 1990). Thus, according to White and Epston (1990), the therapy provides an opportunity for individuals to achieve a new sense of coherence by changing the dominant meaning system and moving toward the construction of novelties. In an attempt to capture this phenomenon, Gonçalves and colleagues (2011) constructed the IMCS, which focuses on the movement of self-reconstruction. This system proposes seven different categories of novelties (or IMs) that occur throughout the therapeutic process as the client progresses from the dominant and problematic meaning system to an alternative, more adjusted one: Actions 1 and 2 (previously termed performing change), Reflections 1 and 2, Protests 1 and 2, and Reconceptualization (see Table 1 for a detailed description of each category and specific clinical examples taken from one of the group counseling sessions implemented during the INSTALL Project).

Action 1 refers to specific actions that challenge a problematic narrative, whereas Action 2 refers to change through new future plans. Reflection 1 is a new form of understanding the problematic narratives and their implications in the client's life, whereas Reflection 2 refers to feelings and thoughts on clients' change. Protest 1 contains a position of critique against the problematic narratives, whereas Protest 2 represents the emergence of new assertive and empowering positions. Reconceptualization is a meta-reflective process that presents a contrast between past and present positions and describes the processes by which changes have been occurring.

As this is the first study that applies IMCS to a group counseling, a different IMCS coding that distinguishes between individual and group IMs was performed and it will be presented in detail in the procedure section.

Previous research using the IMCS in different therapeutic modalities and samples (e.g., Alves et al., 2014; Gonçalves, Mendes, Ribeiro, Angus, \& Greenberg, 2010; Matos, Santos, Gonçalves, \& Martins, 2009) showed that this is a reliable and feasible method for tracking change in psychotherapy. There are several main findings from these studies: (a) Successful psychotherapy involves more time spent in the elaboration of IMs; (b) high-level IMs are more typical of successful psychotherapy, whereas low-level ones dominate unsuccessful cases; and (c) successful psychotherapy usually involves the elaboration of Reconceptualization IMs during the middle of psychotherapy, and they often become dominant IMs as therapy concludes.

We examine possible changes in group counseling by tracking IMs in the NMP. Through this analysis, our goal is to understand how these innovations may constitute opportunities of narrative 


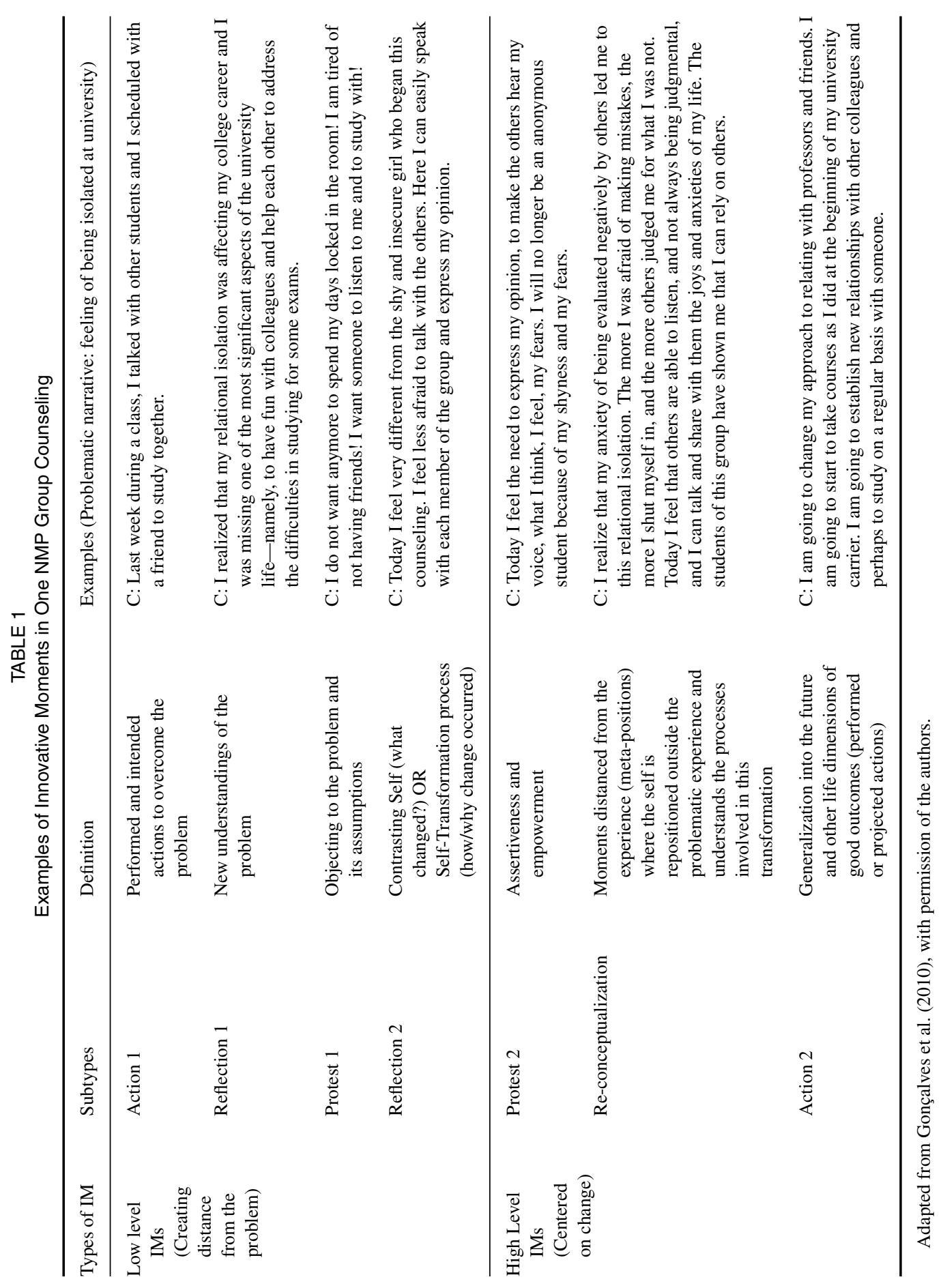


discontinuity, especially regarding the dominant meaning processes brought by students to the counseling sessions. Although the IMCS has recently been applied to the study of the change process in career counseling interventions (Cardoso, Gonçalves, Duarte, Silva, \& Alves, 2014a, 2014b, 2016), this study is the first to use the IMCS in a group counseling environment.

\section{Research Aims}

The aim of this study is to analyze how markers of narrative change (IMs) evolve during the four narrative modes of the counseling process and the follow-up session. The two research questions are as follows: What are the patterns of narrative innovation in NMP and how do they progress throughout the four modes (metaphoric, iconographic, writing, and bodily)? And what are the patterns of narrative innovation during the follow-up session?

A secondary aim is to test the feasibility and acceptability of the NMP counseling intervention based on the outcomes measures used.

\section{METHOD}

\section{Participants}

The group comprises seven students (females $=5$; males $=2$ ) enrolled in the economics degree course at the University of Naples Federico II, Italy. The mean age of the students was 27.86 years $(S D=7.06)$. Five students were taking longer than usual to complete their master's degree, one was enrolled in the third year, and another was enrolled in the second year of the bachelor's degree program. All of the students were considered underachievers as, at the time of their enrollment in the INSTALL project, they had a grade point average (GPA) of less than 23. On a 0 to 30 point scale, a score from 0 to 17 is considered not passing the examination, whereas 18 to 30 corresponds to an adequate performance. Moreover, a GPA between 18 and 23 is usually considered as a low GPA, whereas 24 to 26 is considered a medium GPA, and 27 to 30 represents a high GPA. Moreover, participating students had gained less than 50\% in the European Credit Transfer System (ECTS), a standard for comparing study attainment and performance of students in higher education and for facilitating their transfer and progression throughout the European Union. As with many European countries, Italian universities have adopted this standard, which establishes that for successful completion of a bachelor's degree, 180 ECTS credits must be awarded, with 120 ECTS credits for completing master's degree. Specifically, one academic year corresponds to 60 ECTS credits, which are equivalent to 1500 to 1800 hours of study (including classes and independent study), in all countries, irrespective of standard or qualification type.

Moreover, all of the students were blocked from taking examinations. Three students did not pass any exam from one year, and the others had passed only one or two exams in the year before their participation in the INSTALL project.

The group counseling sessions were held from February to April 2014, and the followup session was conducted in February 2015. The sessions were held once a week and lasted approximately 2 hours each. The group counseling was free and students receive no ECTS for their participation in the counseling service. Instead, each student was given a certificate of participation in the INSTALL project. Students were allowed to be absent only once during 
the counseling. Only three students did not participate in one session due to a class conflict; specifically, one student was absent at the second session, another student at the fourth, and another at the fifth. Moreover, only four students participated in the follow-up session, as the other three were not allowed for work reasons.

All of the students signed an informed consent that allowed the researchers to use both the narrative materials and audio recordings from the sessions for training and research purposes.

\section{Participant Recruitment}

Students were recruited through two different promotion channels: The students' institutional mailing list and advertising in the classroom. The first channel included sending an email to students in which general information about the counseling service (objectives, targets, methods, etc.) was given. The email was sent to the institutional address all students release at the moment of their university enrollment in order to receive a variety of announcements regarding educational and supporting initiatives promoted by the University of Naples. Four of the participating students had joined after having received and responded to that email.

The second channel was a promotion method that SInAPSi Center adopted in collaboration with some university departments, which consisted of presenting university services during the classes mostly frequented by students. During the presentation, students were informed of the counseling service and were asked to contact the service to obtain more information or to sign up directly for the counseling. Three students participated in the counseling after attending the service presentation during classroom time.

Through these two channels it was possible to address both underachieving students who regularly attended classes and those students who did not do so for work reasons or other causes.

\section{Counselor}

In this study, the first author, a Ph.D. clinical psychologist and postdoctoral researcher, served as the counselor. To date, she has been working as an NMP counselor for 4 years and has led many group-counseling paths. She contributed to the development and testing of the NMP model.

\section{Procedure}

\section{Outcome Measures}

To assess the efficacy of INSTALL counseling, some ad hoc questionnaires were administered in three evaluation phases (pretest, posttest, and follow up). Specifically, the academic performance scale (APS) was administered in the pretest phase (at the beginning of the NMP's first session) and at the end of the follow-up session. The APS was not administered during the posttest phase, as no change was expected in the ECTS, GPAs, or the number of examinations passed, owing to the relatively short length of the overall counseling period (approximately 2 months). The questionnaire included several questions regarding the year of enrollment, the degree program, the highest and the lowest scores obtained on an examination, the number of failed examinations, and their number in the ECTS, the number of examinations passed, and the GPAs of each student. 
During the posttest phase (at the end of the NMP's seventh session), the satisfaction questionnaire (SQ) was administered, which included questions regarding the students' degree of satisfaction with the intervention. The students were asked to answer, on a 7-point Likert scale (ranging from extremely satisfied to extremely unsatisfied), questions related to several dimensions of the group counseling (i.e., accessibility and cleanliness of the counseling location, availability and courtesy of the counselor, time of the intervention).

For the present study, we will consider the following questions: (a) What is your overall satisfaction with the counseling? (b) How satisfied were you with the usefulness of the counseling in achieving your university objectives? (c) How satisfied were you with the efficacy of the counseling?

\section{The IMCS}

The IMCS is a qualitative method to track narrative novelties (IMs) that are exceptions to a problematic pattern (in this case, underachievement). In this study, seven types of IMs are considered: Actions 1 and 2, Reflections 1 and 2, Protests 1 and 2, and Reconceptualization. Previous research using the IMCS across various clinical problems and therapeutic models reported an average interjudge percentage of agreement ranging from $84 \%$ to $94 \%$, which supports its reliability (Alves, Mendes, Gonçalves, \& Neimeyer, 2012; Gonçalves et al., 2012; Matos et al., 2009; Mendes et al., 2010). Moreover, the Cohen's Kappa ranged from .86 (Mendes et al., 2010) to.97 (Gonçalves et al., 2010). In this study, the IMCS was used to code the seven sessions of the NMP and the follow-up session.

\section{Coders}

The audio records of the seven sessions and the follow-up session were transcribed verbatim in Italian and independently coded by two researchers. The first coder, one of the researchers who contributed to the development of the IMCS, has years of experience regarding psychotherapy and the use of the IMCS. In addition, he speaks and reads Italian fluently. The second coder, who is the Italian NMP counselor, has extensive training in the IMCS, which consists of five steps and involves the coding of problematic self-narratives and IMs (types and length) in training workbooks and psychotherapy sessions. In addition, a senior author of the IMCS served as an auditor during the coding process.

\section{Coding}

The IMCS coding was composed of three steps:

1. The coders read (in sequential order) all of the transcripts from the seven sessions and the follow-up session to obtain a consensual definition of the problematic pattern. Because this study was the first one in a group intervention context, some differences were introduced in the coding of the problems list in order to interpret the changes that occurred during the intervention as the product of the whole group and not only of some speakers becoming more dominant in the group. 
At first, coders obtained a consensual definition for each student's problematic selfnarratives, following the same procedure adopted with individual psychotherapy, and replicated it for the seven participants in this group counseling. Then, in order to understand if it was possible to identify a group problematic narrative shared by all participants, the coders observed whether the individual problematic narratives were similar to or diverse from each other.

2. In order to identify all of the IMs from the problematic group and self-narratives, each session was coded independently by the two coders and in sequential order (Session 1, Session 2, etc.). Then, coders calculated IM proportion in two ways. At first, coders counted the overall proportion of IMs - that is, they identified when each IM started and ended, irrespective of the student who produced the IM. The proportions were computed for each of the seven IM types (and the overall IMs), for the four narrative modes, the entire counseling process, and the follow-up session. Then, in order to understand if the overall IM proportion could be ascribed to all seven students and not only to some of them, coders observed how this proportion was distributed among all of the participants throughout the counseling and the follow-up session. Specifically, coders counted the proportion of IMs each student produced, by dividing the number of IM words produced by a specific student for the total number of IM words.

3. After the identification of an IM, the coders had to define its type (Action 1, Reflection 1, etc.). In order to understand if the variability of the IM type could be ascribed to all of the students and not only to some dominant members of the group, coders observed which type of IMs each student produced, by analyzing if every student had produced each of the displayed IMs at least once. For example, if in the follow-up session Reflection $1 \mathrm{had}$ emerged, coders would have observed if it was produced at least once by every student. The interjudge agreement was calculated (and independently identified by the two judges) as twice the agreed number of IMs divided by the sum of the IM proportions. Every disagreement in the three coding phases was solved by the intervention of the senior author of the IMCS, who served as an auditor. Furthermore, the analysis was based on the coding of Judge 1 to avoid second-coder bias due to her role as counselor.

\section{RESULTS}

\section{Academic Performance Scale}

As shown in Table 2, except for one student, each participant showed an improvement in ECTS gained and number of examinations passed from pretest to follow-up session. GPA, in contrast, remained almost stable, except for a few cases in which there was a small increase.

Moreover, a Wilcoxon test was performed to assess improvement in the academic performance of the seven participating students. From the pretest to the follow-up phase, there was a significant increase in the ECTS $(Z=-2.201 ; p=0.028)$ and the number of examinations passed $(Z=$ $-2.214 ; p=0.027)$. However, there was no increase in the students' GPAs $(Z=-1.461 ; p=$ $0.144)$.

As previously noted, although GPA is an indicator of the quality of the performance and does not influence the graduation of students, for successfully completed studies, ECTS credits 
TABLE 2

ECTS Gained, Number of Examinations Passed, and GPA of Participating Students from Pretest to Follow-Up

\begin{tabular}{|c|c|c|c|c|c|c|}
\hline & \multicolumn{2}{|c|}{ ECTS } & \multicolumn{2}{|c|}{ N. Examination passed } & \multicolumn{2}{|c|}{ GPA } \\
\hline & Pretest & Follow-up & Pretest & Follow-up & Pretest & Follow-up \\
\hline Student 1 & 20 & 20 & 2 & 2 & 20.5 & 20.5 \\
\hline Student 2 & 36 & 60 & 4 & 6 & 21 & 21.75 \\
\hline Student 3 & 40 & 60 & 7 & 9 & 22 & 22 \\
\hline Student 4 & 41.67 & 137 & 8 & 14 & 22 & 22.5 \\
\hline Student 5 & 83.30 & 140 & 14 & 20 & 22.7 & 23 \\
\hline Student 6 & 131 & 150 & 23 & 25 & 22 & 22.8 \\
\hline Student 7 & 150 & 160 & 24 & 27 & 22 & 23 \\
\hline Mean & 71.71 & 103.85 & 11.71 & 14.71 & 21.74 & 22.22 \\
\hline
\end{tabular}

must be awarded by passing a number of examinations that can vary in each students' formative plan. Thus, the findings seemed to express an overall improvement of the ECTS and number of examinations, which ultimately determines students' graduation.

\section{Satisfaction Questionnaire}

Among the various dimensions analyzed by the satisfaction questionnaire, this study reports only the findings related to overall satisfaction, the self-reported efficacy, and the utility of the counseling. Regarding the satisfaction with the group counseling, all of the group members acknowledged their overall satisfaction ( $N=5$ extremely satisfied; $N=2$ very satisfied). Moreover, five students were extremely satisfied with the efficacy of the intervention and two students were very satisfied. A similar trend was found for the utility of the counseling ( $N=5$ extremely satisfied; $N=2$ very satisfied).

\section{IMCS}

\section{1) Patterns of Narrative Innovation in NMP and their Progress throughout the Four Modes (Metaphoric, Iconographic, Writing, and Bodily).}

At the individual level, some of the problematic narratives are as follows: difficulty integrating different aspects of the student's role (e.g., "Metaphorically speaking, I was referring to these two types of students. One is the student who studies a lot and who can prepare an exam in 20 days; the other is the so-called sloppy student. How can I to merge these two types?"); resentment toward the university (e.g., "My underachieving problems are caused by the university, which, in all honesty, does not work. The professors do not understand us, the programs are too complicated, and there are frequently real idiots as professors!'); fear of disappointing significant others (e.g., "Because I do not want to disappoint them, I do not tell those who are close to me about my poor results on the examinations").

At the group level, the two coders obtained a consensual definition of the problematic group narrative (feeling of inefficacy in performing at the university) that was shared by all of the group 
members and strictly related to their participation in the group counseling. This means that the specific context of counseling for underachieving students oriented the dominant self-narratives of the entire group. Some of the student narratives helped consensually define the problematic group narrative (e.g., "Every university student aims to get 30/30 on the exam, but I do not because I know I'm not capable" and, "I cannot study because I have a mental block and anxiety every time I open the book").

Across the seven sessions, $15.80 \%$ of the entire narrative discourse was devoted to IMs. These may be conceived as both exceptions to the problematic narrative of the group and the individual problematic narratives. The percentage agreement regarding the number of words coded as belonging to IMs (independently of the specific type) was $83.44 \%$.

The proportion of IMs each student produced throughout the counseling oscillated from $8.15 \%$ to $25.06 \%$ of the overall IMs proportion. This means that, although there was not an even distribution of IM proportions among all seven students, each of them produced IMs and contributed to the overall IM proportion.

Overall, the findings suggest that, during the counseling, there were some markers of change, and the students produced IMs with regard to their university experience. The proportion of IMs for each mode presented essentially the same percentages in the first two modes (metaphoric: $14.46 \%$; iconographic: $14.93 \%$ ). In addition, although they decreased in the writing mode $(9.3 \%)$, they increased in the bodily mode to the highest percentage $(24.57 \%)$.

Concerning IM types, reliability for the specific types of IMs assessed by Cohen's Kappa was .94 , thus showing a strong agreement between the judges. Specifically, the most common IM was Reconceptualization (9.4\%), followed by Reflection 2 (6.92\%) and Reflection 1 (5.31\%). The other IMs have a low proportion; Protest 2 was $0.73 \%$, Protest 1 was $0.48 \%$, Action 1 was $0.06 \%$, and Action 2 was absent. Moreover, every student produced, at least once, each of these types of IMs. Thus, all of the students contributed to each type of IM that emerged throughout the counseling. Overall, these findings suggest that the group mostly produced IMs of the reflexive type, especially Reconceptualization; moreover, the group assumed a reflexive position on both problematic self-narratives (Reflection 1) and the process of change (Reflection 2).

Considering the higher proportion of Reflections 1 and 2 and Reconceptualization, only these IMs will be considered in the following process analysis. As shown in Figure 1, Reflections 1 and 2 and Reconceptualization had a different trend across the four narrative modes.

Reflection 1 was the most common IM during the metaphoric mode and had the highest proportion $(11.84 \%)$. However, it started to decrease in the iconographic mode $(6.23 \%)$ and even more in the writing $(4.11 \%)$ and bodily $(2.73 \%)$ modes. The group members, in the first phase of counseling, were mainly involved in a reflection process regarding their difficulties and problems encountered during their university experience. In addition, they produced thoughts, feelings, and intentions that challenged the group and individual problematic narratives.

The following is a clinical vignette of a student during the metaphoric mode:

I realize that the degree is something I want to achieve, it is something for me. I work in order to help my family because my father is unemployed and we are a family of five, which is not easy. My motto is, "Try and try again." I have chosen it because, even if I need 10 or 15 years, I know that I will be able to complete the degree. (Reflection 1, Student 1)

Reflection 2 had a low proportion in the metaphoric mode (1.53\%). However, it reached the highest percentage during the iconographic mode (22.42\%), decreased in the writing mode 


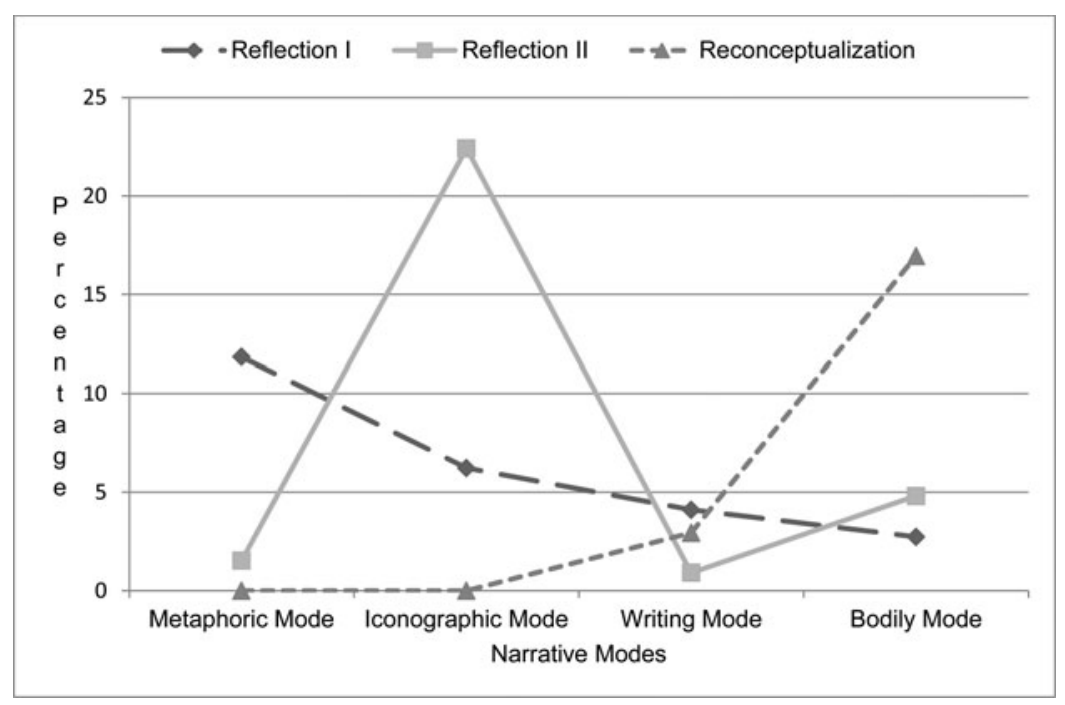

FIGURE 1 Proportion of Reflection I, Reflection II, and Reconceptualization throughout the four narrative modes.

$(0.91 \%)$, and increased again in the bodily mode $(4.81 \%)$. Thus, during the iconographic mode, the students started reflecting on their change and the consequences of such change in their university life. For example:

The quality that I recognize having today is determination. I had it earlier, but I did not recognize it. Now, after listening to them [the group members], I realized that I have determination! I demonstrated that yesterday and I am just now realizing it. (Reflection 2, Student 2)

Reconceptualization is absent in the metaphoric and iconographic modes. It appeared in the writing mode $(2.94 \%)$ and it reached the highest percentage in the bodily mode $(16.95 \%)$. At the end of the counseling, the students were able to describe some form of contrast between the present and the past as well as understand the process that allowed the change. According to one student:

When I started counseling, I spoke just a little and my voice was shaking. I believed that I was inferior to the others. Oh, I see myself changed now, and I approach my studies differently. After we used my vignette titled, "What Anxiety I Have!"-which referred to my anxiety and fear of failure-I no longer had such feelings. Uh, I see myself very changed. However, thanks to them [the group members], they helped me believe in myself. (Reconceptualization, Student 3)

\section{2) Patterns of Narrative Innovation During the Follow-up Session.}

During the follow-up session, the IM proportion reached 39.10\%. The findings suggest that, even a year after the end of counseling, the students continued to produce narrative innovations in regard to their university experience. The proportion of IMs each student produced throughout the follow-up session oscillated from $21.78 \%$ to $28.38 \%$. Despite some oscillations, each student produced IMs and contributed to the overall group proportion. 
The follow-up session was primarily devoted to Reconceptualization, which had the highest proportion (32.86\%), followed by Protest 2 (2.75\%), Reflection 1 (1.77\%), Reflection $2(1.24 \%$ ), and Action $1(0.48 \%)$. Again, Action 2 was absent. Moreover, each type of IM was produced at least once by all of the students; thus, every participant contributed to the variability of the IM type that emerged during the follow-up session.

The findings suggest that, during the follow-up session, the students were mainly involved in the most complex type of innovation, and they were able to compare their past and present selves as well as describe the process of change that allowed this transformation.

\section{DISCUSSION}

This study shows that narrative innovations emerged throughout the seven sessions of counseling and that the students continued to produce them during the follow-up session. The markers of change suggest that the counseling supported the students' overall development from having a dominant feeling of inefficacy to perceiving themselves as competent and able to perform effectively. As for the second aim of the study, the results showed a significant increase in the number of examinations passed and the ECTS gained. This suggests that the change occurred both in the students' meaning system and in their academic performance.

Furthermore, the proportion of IMs displayed a different trend across the four narrative modes and during the follow-up session. Although it reached almost the same percentage in the first two modes (metaphoric and iconographic), it decreased in the writing mode and achieved its highest percentage in the bodily mode. This may constitute a more fluctuating pattern than the one found in individual psychotherapy previously coded with the IMCS, in which (despite some oscillation) the proportions of the IMs tend to present a more continuous progression along the process (Matos et al., 2009).

Despite the present study being the first to apply the IMCS to change in group counseling, some explanatory hypotheses are suggested based on the specificity of the modes and the group setting. For example, the writing mode mainly centered on the narrative description of the university experience-that is, all students have to read what they wrote and expand their discourse by presenting other episodes of their university experience. It is possible to state that, even though the students were asked to elaborate on their university experience, they were more involved in a process of symbolization rather than the elaboration of new meanings. In comparison with the previous modes, the bodily mode was mainly dedicated to the narration of what changed in the students based on their participation in group counseling. The same was found for the follow-up session, in which descriptive narratives were less required and the students were invited to evaluate the reconstruction of their experience. The metaphoric and iconographic modes, however, seemed to follow the same trend in terms of IMs. This most likely occurred because they maintained the same balance in providing descriptive and more elaborative narratives. As noted earlier, during the first two narrative modes, the students were asked to narrate their university experience in a generalized or a projective manner. Thus, it is possible to conclude that both the metaphoric and iconographic modes present an early opportunity to assess the representative function of one's university experience by asking students to not "reveal" themselves but to implicitly narrate their university stories. 
Another explanatory hypothesis concerns the difference between group and individual intervention. Unlike in the individual setting, in group counseling different members can participate in the narrative discourse and with diverse levels of involvement in the elaboration of new meanings regarding their university experiences. Moreover, because each session was mainly devoted to the university stories of one or two group members, these members will probably contribute less in the subsequent sessions, as other members will be asked to narrate their stories. Consequently, it is difficult to expect a linear progression of IMs due to the variability of the students' participation in the group discourse. This does not occur during the bodily mode, when all of the students have narrated their university stories and are asked to elaborate on them through a group sculpture.

Concerning IM types, the most common was Reconceptualization, during both the counseling and the follow-up session. In this case, the counseling mainly promoted the most complex form of narrative innovation. This indicates that students not only can compare their past and present selves but also can explain the process that was involved in their transformations. This is a positive trend similar to the pattern found in previous studies of psychotherapy with good outcomes (Gonçalves, Matos, \& Santos, 2009) or in other studies with nonclinical populations (e.g., Meira, Gonçalves, Salgado, \& Cunha, 2009) in which Reconceptualization emerged in successful groups and may be linked with the magnitude of change over time. Nevertheless, it is also an unexpected trend, because it is different from the one found in previous studies that applied IMCS to counseling interventions with nonclinical population, such as life design counseling (LDC) or career construction counseling (CCC; Cardoso et al. 2014a, 2014b, 2016), in which Reconceptualization did not emerge or presented a very low proportion. We speculate that the different findings may be ascribed to the diverse type of counseling. For example, differently than LDC or CCC, NMP counseling comprises more sessions and is aimed to foster different forms of reflexive process. In other words, Reconceptualization (such as Reflections 1 and 2) is conceived in NMP counseling as a necessary condition for change; thus, students are constantly asked to reflect on the three components that Reconceptualization implicitly contains: (a) the past self, (b) the present self, and (c) the observing position that can assess the change process and describe the transformation from the past to the present (Gonçalves et al., 2012). Moreover, it is interesting to note that, as in LDC and CCC, in the NMP counseling Reconceptualization emerged in the follow-up session when the clients were invited to elaborate on their change process. Specifically, in the NMP counseling, Reconceptualization reached the highest proportion during the follow-up session. This leads us to speculate not only that students continued to elaborate on their change (from an observing position) one year after the end of the counseling but also that Reconceptualization may expand and consolidate when a longer period has passed and students are more prone to reflect on their change as a result of the counseling intervention.

Moreover, during the counseling process, reflexive IMs-such as Reconceptualization and Reflections 1 and Reflection 2-had a higher proportion in comparison with the other IMs (Protests 1 and 2, and Action 1). These findings suggest that the intervention mostly promoted narrative innovations of a reflexive nature. This is consistent with the specificity of this method of intervention, which aims to promote reflexive processes of different complexities rather than supporting students in critiquing their university problems or planning new actions.

Furthermore, Actions 1 and 2 and Protests 1 and 2 mainly involved an agentive stance about one's problems. The ability to act in a transformative manner and be an agent of one's life is the outcome (although not the objective) of the NMP counseling process. The emergence of these "agentive" IMs during the counseling and follow-up session suggests that students can gain 
the ability to assume a proactive and dynamic position in the university context. Nevertheless, their low proportion may be interpreted in two ways: On one hand, it may indicate that the agentic ability is still in its infancy and the reflexive change has not yet been transformed into a strong agentic stance; on the other, we can speculate that students' agency occurs outside the counseling, as reflected in the outcome measures that demonstrated that students were able to gain more ECTS and to pass more examinations. In other words, the reflexive component of students' narratives emerged more throughout the counseling as they were constantly asked to reflect on their university problems and the changes that occurred, whereas discussion of the sphere of action, of what they did and perform outside the intervention, was not requested explicitly.

With regard to the progression of reflexive IMs in the process, the findings show that Reflection 1 had the highest proportion in the metaphoric mode. At the beginning of the counseling, the students mainly reflected on the difficulties they encountered during their university experience and a new comprehension of their problems, causes, and consequences. However, Reflection 1 had the highest proportion in the iconographic mode, showing that (in this phase of group counseling) the students started to mainly reflect on their change and the consequences of such change in their lives. Overall, these findings show the "dialogue" between the two different reflexive positions that were dominant in the two phases and progressively alternated through the process. That is, although the past self-narrative centers on the continuing problem, it gradually leaves room for a new self-narrative that considers the strategies implemented to overcome the problem and reflect on any changes.

Reconceptualization appears during the writing mode, it reaches the highest proportion in the bodily mode, and it is the most common IM during the follow-up session. It is interesting to note that, despite the lowest overall proportion, the writing mode is where Reconceptualization appears. Again, this may depend on the specificity of the mode in which the students are asked to explicitly elaborate on their previous university actions. As noted, the writing mode favors a more subjective process, which may contribute to gaining access to further complex and elaborated thought (Freda, Esposito, \& Quaranta, 2015), even if it occurs at a rudimentary level. The subjectivation process seems to be amplified during the bodily mode and the follow-up session, when all of the students were asked to elaborate on changes in their experiences, reflexively return to them, and compare their past and present selves to describe the process that allowed the transformation. Starting from the writing mode, the students developed a new "shape of thought," a form of meta-reflection (Dimaggio, 2006) that will consolidate and become predominant in the subsequent phase and in the follow-up phase. Furthermore, the students gained access to a more complex narrative structure for change, which allowed them to create a sense of self-continuity based on the integration of their new view of themselves in the context of their problematic self-narratives.

\section{LIMITATIONS AND FUTURE DIRECTIONS}

The main limitation of this study is that it was a pilot study based on a single case of group counseling. More research is necessary to ensure that these results can be replicated in other group case studies. In addition, future research should focus on comparing various counseling groups in terms of academic performance (e.g., high-performing students and low-performing 
ones) to understand the differences in producing narrative innovations between students who benefit from the NMP counseling method and those who do not.

Moreover, because the second coder was the counselor and thus was aware of the status of the case (in terms of both the change process and academic performance improvement), the analysis was based on the coding of the first judge, after which the second judge's coding was only performed to assess reliability and reduce the possibility of bias. In fact, the high degree of agreement between the coders and the auditor reduced the possibility of bias owing to the second coder's expectations. Nevertheless, future research should consider group counseling led by counselors who are not involved in the research process and who are unaware of the status of the case. Consequently, this can help researchers determine whether the patterns observed in this pilot study can be generalized to other cases, in which the researcher serves as the counselor or additional studies in which clinicians adopt the NMP model.

\section{CONCLUSION AND IMPLICATIONS}

This study presented a group counseling model aimed at promoting reflexive processes by engaging underachieving university students in different narrative modes (metaphoric, iconographic, writing, and bodily). The relevance of narrative approaches is not new in the field of counseling (Cochran, 1997; Savickas, 2005), and the use of narratives is widely conceived as a way of understanding how people construct their lives and make sense of their experiences (Bruner, 1990; De Luca Picione \& Freda, 2016; Martino \& Freda, 2016). The NMP model is rooted in the field of narrative theory and specifically addresses the issue of the multimodal narrative approach in amplifying the reflexive construction of meaning (Dicks, Soyinka, \& Coffey, 2006; Pink, 2011).

We examined this process of meaning construction through NMP group counseling sessions and during the follow-up session. By using the IMCS, an interesting perspective of change was offered by tracking narrative innovations along the process. The study showed that, overall, the counseling intervention helped the students develop innovations mainly of a reflexive nature and of a different complexity. This is consistent with the theoretical framework of the INSTALL Project, which is aimed at promoting reflection and reflexivity processes. As this is the first study that applied IMCS to the study of NMP counseling, we can only speculate about how the emerging reflexive IMs may be considered as expressions of reflection and reflexivity processes. More studies are needed in order to understand how these semiotic innovations can overlap; at the moment, we can only hypothesize that Reflection 1 and 2 may be conceived as an expression of reflection, because they can both be conceived as a semiotic innovation about the self, whereas reconceptualization could be seen as a form of reflexivity, as it contains a new understanding of the reasons that allow a subject to recognize the active role assumed in orienting his or her behavior in a context. It was shown the development of reflexive processes with underachieving students was useful in encouraging their learning processes and positively impacting their academic performance. Underachievement among university students is a problem that has significant personal and societal consequences (Balduf, 2009). Moreover, there is an increasing need to provide better-focused guidance, dedicated support and innovative counseling for students to complete higher education (Scheel, Madabhushi, \& Backhaus, 2009). The NMP counseling model responds to this challenge, and the preliminary findings presented here are encouraging. Nevertheless, additional studies are required to determine whether the model is useful with other 
underachievers, and whether the development of reflexive processes may equate to an increase in academic performance for other groups.

It is risky to make inferences from a single case study. However, it is possible to provide some speculative hypotheses to improve the clinical practice of counselors who may adopt the NMP counseling model. For example, despite reconceptualization emerging during the writing mode, in this phase of the counseling there was the lowest proportion of IMs. To balance the descriptive and elaborative levels in the students' narratives, other techniques could be applied during the writing mode. For instance, the counselor could pose specific questions/interventions that foster counterfactual thinking. By inserting an unexpected element (e.g., "What would have happened if ..."), the counselor could redirect students to think about their experiences from a different perspective.

Moreover, the counseling model in this study seems to promote IMs mainly of a reflexive nature, whereas other IMs that involve an agentive stance are less or not represented. The outcome of the counseling path allows students to become agents of their university experience-that is, to gain access to the reversibility of choices to perform effectively at the university (Caston, 2011). In terms of implications, this means rethinking some aspects of the method and inserting particular counseling techniques that can enable students to describe if they have assumed a more agentive stance by performing new actions from one session to another or dealing with their university problems. Specifically, the bodily mode can end by asking students to write a future plan regarding what they are willing to do in following year to overcome the problems. In the same manner, during the follow-up session it could be useful to examine whether students have implemented the planned actions and to question them about the impact of such changes on their university experiences.

\section{FUNDING}

This work was made possible by a grant from the European Commission (Grant Agreement 20114040 Project 517750-LLP-1-2011-1-IT-ERASMUS-ESIN). This work was also made possible due to funding from the Short International Mobility Program in the International Relations Office of the University of Naples Federico II, Italy.

\section{NOTE}

1. This project was funded with support from the European Commission. This publication reflects the views of the author, and the European Commission cannot be held responsible for any use that may be produced from the information contained herein.

\section{ORCID}

Miguel M. Gonçalves ๑ http://orcid.org/0000-0003-2575-7221 


\section{REFERENCES}

Adler, J. M., Skalina, L. M., \& McAdams, D. P. (2008). The narrative reconstruction of psychotherapy and psychological health. Psychotherapy Research, 18, 719-734. http://dx.doi.org/10.1080/10503300802326020

Alves, D., Fernández-Navarro, P., Baptista, J., Ribeiro, E., Sousa, I., \& Gonçalves, M. M. (2014). Innovative moments in grief therapy: The meaning reconstruction approach and the processes of self-narrative transformation. Psychotherapy Research, 24(1), 25-41. http://dx.doi.org/10.1080/10503307.2013.814927

Alves, D., Mendes, I., Gonçalves, M. M., \& Neimeyer, R. A. (2012). Innovative moments in grief therapy: Reconstructing meaning following perinatal death. Death Studies, 36, 795-818. http://dx.doi.org/10.1080/07481187.2011.608291

Angus, L., Levitt, H., \& Hardtke, K. (1999). The narrative processes coding system: Research applications and implications for psychotherapy practice. Journal of Clinical Psychology, 55, 1255-1270. http://dx.doi.org/10.1002/(SICI)10974679(199910)55:10<1255:AID-JCLP7>3.0.CO;2-F

Bacchini, D., Esposito, G., \& Affuso, G. (2009). Social experience and school bullying. Journal of Community \& Applied Social Psychology, 19(1), 17-32. http://dx.doi.org/10.1002/casp.975

Balduf, M. (2009). Underachievement among college students. Journal of Advanced Academics, 20(2), $274-294$. http://dx.doi.org/10.1177/1932202×0902000204

Baslanti, U., \& McCoach, D. B. (2006). Factors related to the underachievement of university students in Turkey. Roeper Review, 28(4), 210-215. http://dx.doi.org/10.1080/02783190609554366

Bleakley, A. (2006). From reflective practice to holistic reflexivity. Studies in Higher Education, 24(3), 315-330. http://dx.doi.org/10.1080/03075079912331379925

Bruner, J. (1990). Acts of meaning. Cambridge, MA: Harvard University Press.

Cardoso, P., Gonçalves, M. M., Duarte, M. E., Silva, J., \& Alves, D. (2016). Life design counseling process and outcome: A case study with an adolescent. Journal of Vocational Behavior, 93, 58-66.

Cardoso, P., Silva, J. R., Gonçalves, M. M., \& Duarte, M. E. (2014a). Innovative moments and change in career construction counseling. Journal of Vocational Behavior, 84, 11-20. http://dx.doi.org/

Cardoso, P., Silva, J. R., Gonçalves, M. M., \& Duarte, M. E. (2014b). Narrative innovation in life design counseling: The case of Ryan. Journal of Vocational Behavior, 85, 276-286. http://dx.doi.org/10.1016/j.jvb.2014.08.001

Caston, J. (2011). Agency as a psychoanalytic idea. Journal of the American Psychoanalytic Association, 59(5), 907-938. http://dx.doi.org/10.1177/0003065111422541

Cochran, L. (1997). Career counseling. A narrative approach. Newbury Park, CA: Sage.

Council of the European Union. (2013). Council conclusions on the social dimension of higher education. Retrieved from http://www.consilium.europa.eu/uedocs/cms_data/docs/pressdata/en/educ/137144.pdf

De Luca Picione, R., \& Freda, M. F. (2016). Possible use in psychology of threshold concept in order to study sense-making processes. Culture \& Psychology, 22(3), 362-375. http://dx.doi.org/1354067×16654858

Dicks, B., Soyinka, B., \& Coffey, A. (2006). Multimodality ethnography. Qualitative Research, 6(1), 77-96. http://dx.doi.org/10.1177/1468794106058876

Dimaggio, G. (2006). Disorganized narratives in clinical practice. Journal of Constructivist Psychology, 19, $103-108$. http://dx.doi.org/10.1080/10720530500508696

Esposito, G., \& Freda, M. F. (2015). Evaluating training context competence of use: Productive and unproductive models of use. Evaluation and Program Planning, 50, 77-87. http://dx.doi.org/10.1016/j.evalprogplan.2015.02.003

Esposito, G., \& Freda, M. F. (2016). Reflective and agentive functions of narrative writing: A qualitative study on the narratives of university students. Integrative Psychological \& Behavioral Science, 50(2), 333-357. http://dx.doi.org/10.1007/s12124-015-9323-5

Esposito, G., Freda, M. F., \& Bosco, V. (2015). Examining perception of competency through practicum competencies outline. European Journal of Training and Development, 39(8), 700-720. http://dx.doi.org/10.1108/EJTD-05-20150037

Freda, M. F., \& Esposito, G. (In press). Promoting reflection and reflexivity through narrative devices: Narrative mediation path qualitative multimodal method. Qualitative Research Journal, 17(1). doi: 10.1108/QRJ-06-2016-0039.

Freda, M. F., Dicé, F., Auricchio, M., Salerno, M., \& Valerio P. (2014). Suspended sorrow: The crisis in the understanding of the diagnosis for the mothers of children with a disorder of sex development. International Journal of Sexual Health, 27(2), 186-198. http://dx.doi.org/10.1080/19317611.2014.957793

Freda, M. F., Esposito, G., \& Quaranta, T. (2015). Promoting mentalization in clinical psychology at universities: A linguistic analysis of student accounts. Europe's Journal of Psychology, 11(1), 34-49. http://dx.doi.org/10.5964/ejop.v11i1.812 
Gonçalves, M. M., Matos, M., \& Santos, A. (2009). Narrative therapies and the nature of "unique outcomes" in the construction of change. Journal of Constructivist Psychology, 22, 1-23.

Gonçalves, M. M., Mendes, I., Cruz, G., Ribeiro, A. P., Angus, L., \& Greenberg, L. (2012). Innovative moments and change in client-centered therapy. Psychotherapy Research, 22, 389-401. http://dx.doi.org/10.1080/10503307.2012.662605

Gonçalves, M. M., Mendes, I., Ribeiro, A. P., Angus, L. E., \& Greenberg, L. S. (2010). Innovative moments and change in emotion-focused therapy: The case of Lisa. Journal of Constructivist Psychology, 23(4), 267-294. http://dx.doi.org/10.1080/10720537.2010.489758

Gonçalves, M. M., Ribeiro, A. P., Mendes, I., Alves, D., Silva, J., Rosa, C., Batista, J., Fernández-Navarro, P., \& Braga, C. (In press). Innovative moments, ambivalence and ambivalence resolution: Coding systems and main findings. Psychotherapy Research.

Gonçalves, M. M., Ribeiro, A. P., Mendes, I., Matos, M., \& Santos, A. (2011). Tracking novelties in psychotherapy process research: The innovative moments coding system. Psychotherapy Research, 21, 497-509. http://dx.doi.org/10.1080/10503307.2011.560207

Kember, D., McKay, J., Sinclair, K., \& Wong, F. K. Y. (2008). A four-category scheme for coding and assessing the level of reflection in written work. Assessment \& Evaluation in Higher Education, 33(4), 369-379. http://dx.doi.org/10.1080/02602930701293355

Kress, G. (2005). Gains and losses: New forms of texts, knowledge, and learning. Computers and Composition, 22(1), $5-22$.

Lieblich, A. (2012). Healing plots: Writing and reading in life-stories groups. Qualitative Inquiry, 19(1), 46-52. http://dx.doi.org/10.1177/1077800412462982

Martino, M. L., \& Freda, M. F. (2016). Post-traumatic growth in cancer survivors: Narrative markers and functions of the experience's transformation. The Qualitative Report, 21(4), 765-780. http://nsuworks.nova.edu/tqr/vol21/iss4/11

Matos, M., Santos, A., Gonçalves, M. M., \& Martins, C. (2009). Innovative moments and change in narrative therapy. Psychotherapy Research, 19, 68-80. http://dx.doi.org/10.1080/10503300802430657

McAdams, D. P., Bauer, J. J., Sakaeda, A. R., Anyidoho, N. A., Machado, M. A., Magrino-Failla, K., White, K. W., \& Pals, J. L. (2006). Continuity and change in the life story: A longitudinal study of autobiographical memories in emerging adulthood. Journal of Personality, 74(5), 1371-1400. http://dx.doi.org/10.1111/j.1467-6494.2006.00412.x

Meira, L., Gonçalves, M. M., Salgado, J., \& Cunha, C. (2009). Everyday life change: Contribution to the understanding of daily human change. In M. Todman (Ed.), Self-regulation and social competence: Psychological studies in identity, achievement, and work-family dynamics (pp. 145-154). Athens, Greece: ATINER.

Mendes, I., Ribeiro, A. P., Angus, L., Greenberg, L. S., Sousa, I., \& Gonçalves, M. M. (2010). Narrative change in emotion-focused therapy: How is change constructed through the lens of the innovative moments coding system? Psychotherapy Research, 20(6), 692-701. http://dx.doi.org/10.1080/10503307.2010.514960

Moore, T. (2011). Critical thinking: Seven definitions in search of a concept. Studies in Higher Education, 36, 1-17. http://dx.doi.org/10.1080/03075079.2011.586995

Murdock, N. L., Dual, C., \& Nilsonn J. E. (2012). Emerging approaches to counseling intervention. Theory, research, practice, and training. The Counseling Psychologist, 40(7), 966-975. http://dx.doi.org/10.1177/0011000012460663

Muskens, G. (2011). Inclusion and education in European countries: Methodological considerations. Quality and Quantity, 47(1), 237-255. http://dx.doi.org/10.1007/s11135-011-9514-1

Padykula, L. N., \& Horwitz, M. (2011). Using psychodynamic concepts to measure interpersonal competencies during social work training. International Journal of Applied Psychoanalytic Studies, 9(1), 48-61. http://dx.doi.org/10.1002/aps

Pink, S. (2011). Multimodality, multisensoriality, and ethnographic knowing: Social semiotics and the phenomenology of perception. Qualitative Research, 11(3), 261-276. http://dx.doi.org/10.1177/1468794111399835

Radman, Z. (1997). Metaphors: Figures of the mind. Dordrecht, The Netherlands: Kluwer Academic.

Reis, S. M., \& McCoach, D. B. (2000). The underachievement of gifted students: What do we know and where do we go? Gifted Child Quarterly, 44, 152-170.

Richardson, M. S. (2012). Counseling for work and relationship. The Counseling Psychologist, 40(2), 190-242. http://dx.doi.org/10.1177/0011000011406452

Rimm, S. B. (2003). Underachievement: A national epidemic. In N. Colangelo \& G. A. Davis (Eds.), Handbook of gifted education (pp. 424-443). Boston, MA: Allyn \& Bacon.

Salvatore, S. (2011). Psychotherapy research needs theory. Outline for an epistemology of the clinical exchange. Integrative Psychological and Behavioral Science, 45, 366-388. http://dx.doi.org/10.1007/s12124-011-9180-9

Savickas, M. L. (2005). The theory and practice of career construction. In S. D. Brown \& R. W. Lent (Eds.), Career development and counseling (pp. 42-70). Hoboken, NJ: Wiley. 
Scheel, M. J., Madabhushi, S., \& Backhaus, A. (2009). The academic motivation of at-risk students in a counseling prevention program. The Counseling Psychologist, 37(8), 1147-1178. http://dx.doi.org/10.1177/0011000009338495 Shaw, M. A. (2002). The object relations technique: Assessing the individual. Manhasset, NY: ORT Institute.

Taylor, C. A. (2011). More than meets the eye: The use of video narratives to facilitate doctoral students' reflexivity on their doctoral journeys. Studies in Higher Education, 36(4), 441-458. http://dx.doi.org/10.1080/03075071003714115

Twemlow, S., Fonagy, P., \& Sacco, F. C. (2005). A developmental approach to mentalize communities: The peaceful schools experiment. Bulletin of the Menninger Clinic, 69(4), 282-304. http://dx.doi.org/10.1521/bumc.2005.69.4.282

Vallario, L. (2012). La scultura della famiglia [Family sculpture]. Milan, Italy: Franco Angeli.

White, M., \& Epston, D. (1990). Narrative means to therapeutic ends. New York, NY: Norton. 